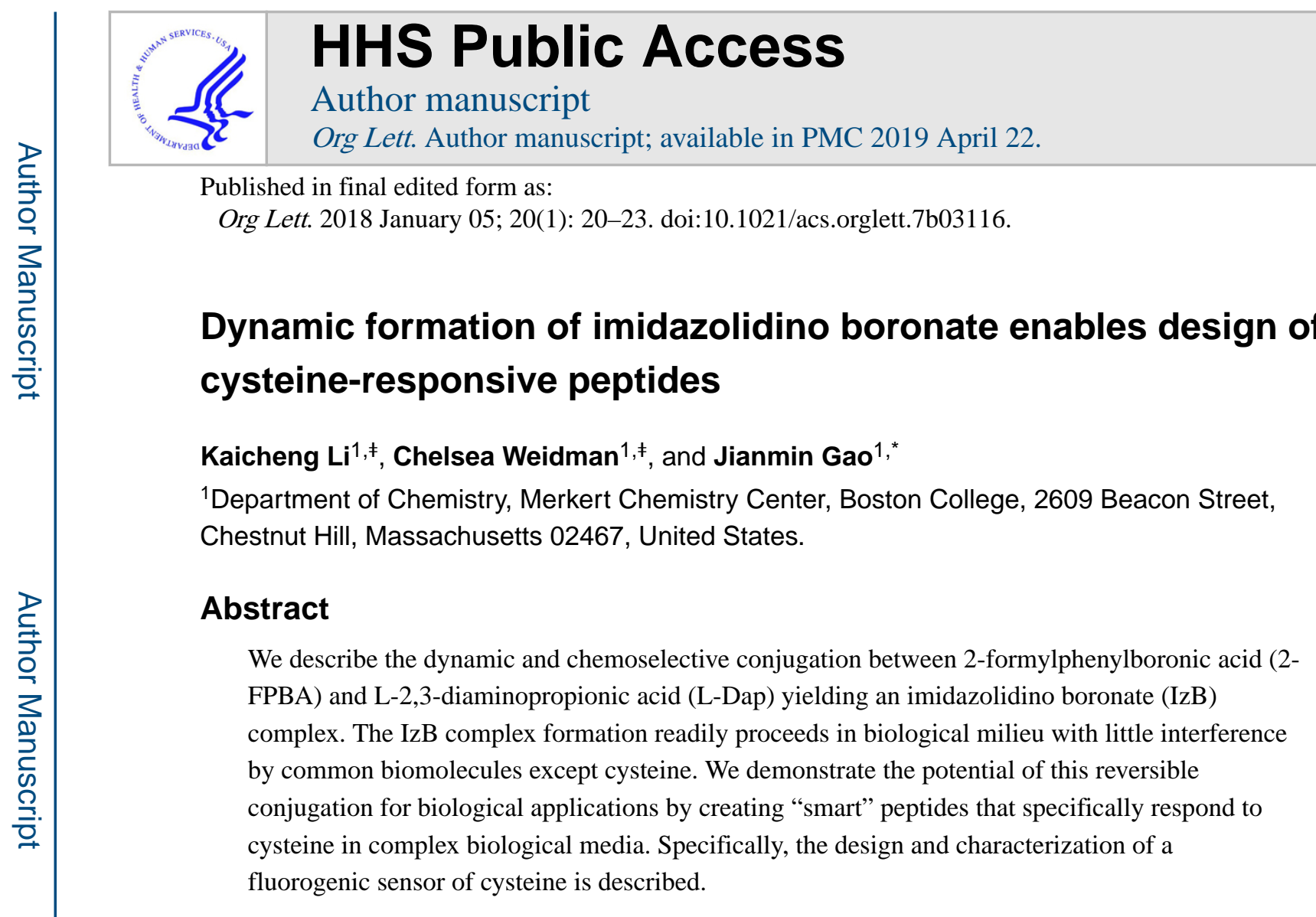

\title{
Graphical Abstrct
}

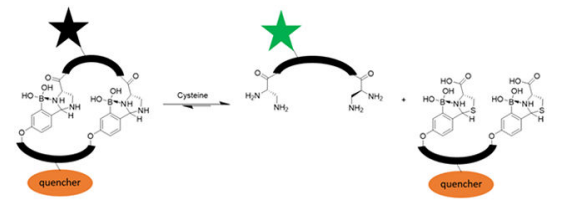

Reversible conjugation reactions that readily proceed in biological media are rapidly gaining attention, not only in dynamic combinatorial chemistry, ${ }^{1}$ but also in ligand design to target biomolecules. $^{2}$ The dynamic formation of boronate esters ${ }^{3-5}$ has been extensively investigated in the design of molecular probes for proteins, nucleic acids and carbohydrates and has given rise to the anticancer drug bortezomib. ${ }^{6}$ Similarly, the reversible Michael addition of thiols has been devised to enable covalent kinase inhibitors, ${ }^{7,8}$ and more recently sub-stoichiometric sensors of glutathione. ${ }^{9}$ Exploiting the reactivity of the amino groups in biomolecules, we and others have demonstrated the use of iminoboronate chemistry (Figure 1A) to facilitate binding to proteins and lipids. ${ }^{10-12}$ Collectively these examples showcase the power of reversible covalent binding mechanisms in molecular recognition.

This contribution describes a new dynamic conjugation reaction, in which 2formylphenylboronic acid (2-FPBA) reversibly conjugates with 1,2-diamines to give

\footnotetext{
*Corresponding Author: jianmin.gao@bc.edu.

${ }^{\ddagger}$ These authors contributed equally to this work.

Supporting Information

Additional NMR and X-ray data of the IzB complex and KL21; detailed experimental protocols; small molecule and peptide synthesis.

The Supporting Information is available free of charge on the ACS Publications website.
} 
imidazolidino boronates (IzB). As a part of our studies of iminoboronate formation of amino acids, we recently reported the fast and chemoselective conjugation between 2-FPBA and cysteines. ${ }^{13}$ This conjugation proceeds via an iminoboronate intermediate to give a thiazolidino boronate (TzB) complex (Figure 1B). In comparison to the corresponding thiazolidine, a TzB complex enjoys greater thermodynamic stability due to formation of the N-B dative bond. Similar findings were independently reported by the Gois group around the same time. ${ }^{14}$ Herein, we show that L-2,3-diaminopropionic acid (L-Dap) reacts with 2FPBA via an analogous mechanism to give an IzB complex (Figure 1C). Interestingly, we found the IzB complex formation shows distinct thermodynamic and kinetic properties in comparison to the $\mathrm{TzB}$ analogue.

The IzB complex of 2-FPBA and L-Dap was confirmed by X-ray crystallography (Figure $2 \mathrm{~A}$, Figure S1). X-ray analysis of the crystals obtained from a water/methanol mixture at $\mathrm{pH}$ 7.4 revealed a fused multicyclic structure, featuring an imidazolidine ring that forms an N-B dative bond with the boronic acid moiety. Furthermore, mixed anhydride formation between the - $\mathrm{COOH}$ of L-Dap and the boronic acid affords an additional ring in crystal structure of the IzB complex. Not surprisingly, the IzB crystal structure displays a high degree of analogy to that of the TzB complex formed between 2-FPBA and cysteine. ${ }^{13}$

The IzB complex formation was further characterized via NMR spectroscopy. Mixing 2FPBA and L-Dap at $10 \mathrm{mM}$ concentration resulted in quick and complete disappearance of the aldehyde peak of 2-FPBA at $9.8 \mathrm{ppm}$. Instead, a pair of new peaks emerged at 5.75 and $5.85 \mathrm{ppm}$, which is indicative of imidazolidine formation. The ratio of the two peaks varies with high $\mathrm{pH}$ favoring the peak at $5.75 \mathrm{ppm}$ and low $\mathrm{pH}$ favoring the $5.85 \mathrm{ppm}$ peak (Figure 2B). Similar to the TzB complex, we assign the dominant species at low $\mathrm{pH}$ (5.85 ppm peak) to be the mixed anhydride as revealed by the crystal structure, yet the $5.75 \mathrm{ppm}$ peak corresponds to the hydrolysis (open) product of the mixed anhydride under slightly basic conditions. Different from TzB, an open IzB complex could in principle exist as two isomers with the $\alpha$ - and $\beta$-amine forming N-B dative bond respectively. It is challenging to determine the relative abundance of the isomers which are likely in quick equilibrium to give a single set of ${ }^{1} \mathrm{H}-\mathrm{NMR}$ peaks as seen in Figure 2B ( $\left.\mathrm{pH} 10\right)$.

We note that the IzB complex formation is not limited to L-Dap, but rather applies to 1,2diamines in general (Figure S2). Further analysis of the IzB formation revealed that the conjugation between 2-FPBA and L-Dap is rapidly reversible. Conveniently, 2-FPBA exhibits a characteristic absorption maximum at $254 \mathrm{~nm}$, which drops substantially upon IzB formation. By monitoring the absorption change, we performed a dilution experiment to probe the dissociation kinetics. Upon 16.7x dilution of an IzB stock $(1 \mathrm{mM})$, the dissociation reached equilibrium in less than $30 \mathrm{~s}$ as indicated by the plateau of absorption at $254 \mathrm{~nm}$ (Figure 2C). Fitting the relaxation curve yielded a relaxation time constant of $6.7 \pm 0.3 \mathrm{~s}$, which agrees nicely with the relaxation time constant extracted from the kinetic studies of the forward reaction (Figure S3). With the reversibility established, we performed an equilibrium titration experiment (Figure 2D), which yielded a $K_{d}$ value of $100( \pm 8) \mu \mathrm{M}$. The $K_{d}$ value, in combination with the relaxation time constant, allowed calculation of the forward and backward reaction rate constants, which turn out to be $0.82 \times 10^{3} \mathrm{M}^{-1} \mathrm{~s}^{-1}$ and $0.082 \mathrm{~s}^{-1}$, respectively. The boronic acid-mediated fast conjugation (forward reaction) is 
comparable to that of $\mathrm{TzB}$ formation, as well as the boronic acid-accelerated conjugation reactions of a-nucleophiles. ${ }^{15-18}$ However, the dissociation of the IzB complex is much faster than the TzB analogue. While the IzB complex dissociates over seconds upon dilution to $60 \mu \mathrm{M}$, the dissociation of the TzB complex is not observed even at $10 \mu \mathrm{M}$, which is the detection limit of the UV based analysis. Nevertheless, the TzB complex was found to exchange with an N-terminal cysteine bearing peptide over 1-2 hours, ${ }^{13}$ which suggests the TzB dissociation is much slower than IzB.

Similar to the TzB formation, we anticipate the IzB complex formation to show little interference from abundant biomolecules. Indeed, the addition of 10 equivalents of glucose, serine, lysine, and glutathione resulted in little change of the IzB signature peaks (Figure 3). Not surprisingly, the addition of cysteine does cause the dissociation of the IzB complex via competitive TzB formation. Specifically, the addition of 1 equivalent of cysteine $(1 \mathrm{mM})$ converted $82 \%$ of the IzB complex to the TzB complex. Taking the $K_{d}$ value of IzB formation as a reference, the competitive formation of the IzB and TzB complexes allowed estimation of the $K_{d}$ value for TzB formation to be $4.6 \mu \mathrm{M}$. The low $K_{d}$ value is consistent with the complete conjugation of 2-FPBA and cysteine even at low micromolar concentrations. ${ }^{13}$

The bioorthogonal IzB formation and its facile exchange with cysteine makes it suitable for developing "smart" peptides that sense and respond to cysteine, this important amino acid that plays various roles in biology. For example, abnormal levels of cysteine concentration are associated with many diseases including coronary heart disease. ${ }^{19}$ Furthermore, the $\mathrm{IzB} / \mathrm{TzB}$ exchange should apply to important cysteine derivatives including proteins with $\mathrm{N}$ terminal cysteines, as well as bacillithiol, the redox regulator of Staphylococcus aureus. ${ }^{20}$ "Smart" peptides ${ }^{21-23}$ have been developed to sense enzymes, $\mathrm{pH}$ or redox potential and have shown applications in imaging, hydrogel formation and targeted drug delivery. However, few examples describe peptides that respond to specific small molecule metabolites in complex biological milieu. Herein, we describe two different designs of cysteine-responsive peptides. First, installing an N-terminal L-Dap residue together with a 2FPBA motif yields a cyclic peptide that readily linearizes upon cysteine exposure (Figure 4A). The peptide KL21 was synthesized as a proof-of-concept example and examined for cyclization using ${ }^{1} \mathrm{H}-\mathrm{NMR}$ (Figure $\left.\mathrm{S} 4\right)$. Under strongly acidic conditions $(\mathrm{pH}<2$ ), the peptide exists in the linear form with the signature aldehyde peak observed at $9.8 \mathrm{ppm}$. When the $\mathrm{pH}$ is raised above 5.9 , the peptide is fully cyclized as suggested by the complete disappearance of the aldehyde peak and the appearance of the signature peak of IzB complex at $5.8 \mathrm{ppm}$. Consistent with the bioorthogonality of the IzB formation, the cyclic KL21 exhibits remarkable stability under neutral conditions: the ${ }^{1} \mathrm{H}-\mathrm{NMR}$ signature of the peptide remains essentially unchanged with the addition of serine, glucose, lysine or glutathione (Figure S5). We then examined the response of KL21 to cysteine via a titration experiment (Figure 4C). As expected, the addition of cysteine caused the decrease of the IzB signature peak at $5.8 \mathrm{ppm}$ and the concurrent appearance of the TzB signature peaks at 6.0 and 6.2 $\mathrm{ppm}$. Quantitative analysis based on the integrated peak areas yields an $\mathrm{EC}_{50}$ of $2.0 \mathrm{mM}$ (Figure S6). The cysteine-induced peptide linearization encounters little interference by complex biological media: when prepared in solutions with $10 \%$ fetal bovine serum (FBS), 
KL21 gave a titration curve essentially identical to that obtained without FBS (Figure S7), which nicely showcases the bioorthogonality of the IzB/TzB exchange.

As both the IzB and TzB formation is reversible, we anticipated that KL21 should be able to respond to the dynamic changes of free cysteine concentration in complex biological media. As a proof-of-concept demonstration, we recorded the ${ }^{1} \mathrm{H}-\mathrm{NMR}$ spectra of the KL21/ cysteine/FBS mixture over time. The overlaid spectra show gradual decrease of the TzB peaks and regeneration of the IzB cyclized peptide as a result of cysteine oxidation (Figure S8).

To better facilitate cysteine analysis in complex biological milieu, we sought to develop fluorogenic reporters of cysteine using the IzB/TzB exchange. Disappointingly, incorporating a fluorophore-quencher pair into KL21 failed to give fluorogenic responses to cysteine titration (Figure S9), likely due to insufficient fluorophore-quencher separation even in the linear form of the peptide. Alternatively, we explored IzB-mediated bimolecular association to develop fluorogenic sensors (Figure 4B). The peptides KL22 and KL23 were synthesized to display two copies of L-Dap and 2-FPBA residue respectively. Furthermore, KL22 was labeled with a fluorescein and KL23 was labeled with a dabcyl quencher (Figure S10). The bivalent display of 2-FPBA and L-Dap is expected to give specific and high affinity association of the two peptides. Indeed, a titration experiment monitored by fluorescence quenching (Figure S10) revealed that KL22 associates with KL23 at sub- $\mu$ M concentrations $\left(K_{d^{*}} 0.3 \mu \mathrm{M}\right)$. At such low concentrations, KL22-23 association would not be possible with a single IzB linkage. Instead, they must associate with two IzB linkages to give a cyclic complex. As expected, the KL22-23 complex dissociates with addition of cysteine, leading to strong fluorescence emission (Figure 4D). Fitting the titration curve yields an $\mathrm{EC}_{50}$ value of $0.2 \mathrm{mM}$, which nicely falls into the physiological concentration range of cysteine. ${ }^{24}$ The fluorogenic response is highly specific to cysteine: no fluorescence increase was observed with the addition of glutathione, glucose, serine, or lysine (Figure 4D). Excitingly, similar fluorogenic response to cysteine titration was observed even with the presence of $10 \%$ FBS (Figure S11).

To conclude, this contribution describes the dynamic conjugation between 2-FPBA and LDap leading to the formation of an IzB complex. The IzB conjugation is rapidly reversible under physiologic conditions with an apparent $K_{d}$ of $\sim 100 \mu \mathrm{M}$. Importantly, the IzB formation is chemoselective showing little interference by abundant biomolecules except cysteine. The L-Dap and 2-FPBA combination, when incorporated into peptides, elicits spontaneous and rapid formation of cyclic peptides in either unimolecular or bimolecular fashion. The IzB cyclized peptides display robust stability in blood serum, yet are able to detect free cysteine and report the dynamic change of cysteine concentrations. A number of fluorescence sensors of cysteine have been documented in literature. ${ }^{24-27}$ These previously reported sensors detect cysteine via irreversible conjugation of cysteine to fluorophorederived aldehydes. Most of these conjugation reactions are kinetically slow and often require the use of acidic conditions. In contrast, the IzB/TzB exchange proceeds rapidly under physiological $\mathrm{pH}$. More importantly, the IzB cyclized peptides react with cysteine in a reversible manner. Reversible, thermodynamically controlled sensors of analytes have been 
scarce in literature, ${ }^{9,28}$ however they are desirable as they can be used to monitor the dynamic changes of the analyte in real time.

Although the unimolecularly cyclized peptide failed to give fluorogenic sensors of cysteine, we believe the cysteine-mediated decyclization will enable design of functional molecules in various applications. Furthermore, the dynamic exchange between IzB and TzB should be applicable not only to cysteine, but also to proteins with $\mathrm{N}$-terminal cysteines, as well as small molecules that display 1,2-aminothiol functionalities such as bacillithiol found in bacterial pathogens. ${ }^{20}$ Our ongoing research is extending the IzB/TzB chemistry to detect these important biomolecules.

\section{Supplementary Material}

Refer to Web version on PubMed Central for supplementary material.

\section{ACKNOWLEDGMENT}

We thank Dr. Bo Li for solving the crystal structure of the IzB conjugate between 2-FPBA and L-Dap. The financial support of this work is provided by the National Institutes of Health via grant GM102735 to JG.

\section{REFERENCES}

(1). Cougnon FBL; Sanders JK M. Acc. Chem. Res 2012, 45 (12), 2211.

(2). Bandyopadhyay A; Gao J Curr. Opin. Chem. Biol 2016, 34, 110. [PubMed: 27599186]

(3). James TD; Sandanayake KRAS; Shinkai S Angew. Chem. Int. Ed. Engl 1996, 35 (17), 1910.

(4). Dai C; Sagwal A; Cheng Y; Peng H; Chen W; Wang B Pure Appl. Chem 2012, 84 (11), 2479.

(5). Dowlut M; Hall DG J. Am. Chem. Soc 2006, 128 (13), 4226. [PubMed: 16568987]

(6). Adams J; Kauffman M Cancer Invest. 2004, 22 (2), 304. [PubMed: 15199612]

(7). Bradshaw JM; McFarland JM; Paavilainen VO; Bisconte A; Tam D; Phan VT; Romanov S; Finkle D; Shu J; Patel V; Ton T; Li X; Loughhead DG; Nunn PA; Karr DE; Gerritsen ME; Funk JO; Owens TD; Verner E; Brameld KA; Hill RJ; Goldstein DM; Taunton J Nat. Chem. Biol 2015, 11 (7), 525. [PubMed: 26006010]

(8). Serafimova IM; Pufall MA; Krishnan S; Duda K; Cohen MS; Maglathlin RL; McFarland JM; Miller RM; Frödin M; Taunton J Nat. Chem. Biol 2012, 8 (5), 471. [PubMed: 22466421]

(9). Jiang X; Yu Y; Chen J; Zhao M; Chen H; Song X; Matzuk AJ; Carroll SL; Tan X; Sizovs A; Cheng N; Wang MC; Wang J ACS Chem. Biol 2015, 10 (3), 864. [PubMed: 25531746]

(10). Cal PMSD; Vicente JB; Pires E; Coelho AV; Veiros LF; Cordeiro C; Gois PM P. J. Am. Chem. Soc 2012, 134 (24), 10299.

(11). Bandyopadhyay A; McCarthy KA; Kelly MA; Gao J Nat. Commun 2015, 6, 6561. [PubMed: 25761996]

(12). Akçay G; Belmonte MA; Aquila B; Chuaqui C; Hird AW; Lamb ML; Rawlins PB; Su N; Tentarelli S; Grimster NP; Su Q Nat. Chem. Biol 2016, 12 (11), 931. [PubMed: 27595327]

(13). Bandyopadhyay A; Cambray S; Gao J Chem. Sci 2016.

(14). Faustino H; Silva MJSA; Veiros LF; Bernardes GJL; Gois PM P. Chem. Sci 2016, 7 (8), 5052.

(15). Schmidt P; Stress C; Gillingham D Chem. Sci 2015, 6 (6), 3329. [PubMed: 29142692]

(16). Bandyopadhyay A; Gao J Chem. - Eur. J 2015, 21 (42), 14748. [PubMed: 26311464]

(17). Dilek O; Lei Z; Mukherjee K; Bane S Chem. Commun 2015, 51 (95), 16992.

(18). Bandyopadhyay A; Cambray S; Gao JJ Am. Chem. Soc 2017, 139 (2), 871.

(19). Özkan Y; Özkan E; Şimşek B Int. J. Cardiol 2002, 82 (3), 269. [PubMed: 11911915] 
(20). Newton GL; Rawat M; La Clair JJ; Jothivasan VK; Budiarto T; Hamilton CJ; Claiborne A; Helmann JD; Fahey RC Nat. Chem. Biol 2009, 5 (9), 625. [PubMed: 19578333]

(21). Zhou J; Xu B Bioconjug. Chem 2015, 26 (6), 987. [PubMed: 25933032]

(22). Ye D; Shuhendler AJ; Cui L; Tong L; Tee SS; Tikhomirov G; Felsher DW; Rao J Nat. Chem 2014, 6 (6), 519. [PubMed: 24848238]

(23). Bowerman CJ; Nilsson BL J. Am. Chem. Soc 2010, 132 (28), 9526. [PubMed: 20405940]

(24). Tian M; Guo F; Sun Y; Zhang W; Miao F; Liu Y; Song G; Ho C-L; Yu X; Sun JZ; Wong W-Y Org. Biomol. Chem 2014, 12 (32), 6128. [PubMed: 24991968]

(25). Wang W; Rusin O; Xu X; Kim KK; Escobedo JO; Fakayode SO; Fletcher KA; Lowry M; Schowalter CM; Lawrence CM; Fronczek FR; Warner IM; Strongin RM J. Am. Chem. Soc 2005, 127 (45), 15949. [PubMed: 16277539]

(26). Yang Z; Zhao N; Sun Y; Miao F; Liu Y; Liu X; Zhang Y; Ai W; Song G; Shen X; Yu X; Sun J; Wong W-Y Chem. Commun 2012, 48 (28), 3442.

(27). Tong H; Zhao J; Li X; Zhang Y; Ma S; Lou K; Wang W Chem. Commun 2017, 53 (25), 3583.

(28). Umezawa K; Yoshida M; Kamiya M; Yamasoba T; Urano Y Nat. Chem 2017, 9 (3), 279. [PubMed: 28221345] 
A Previous Work

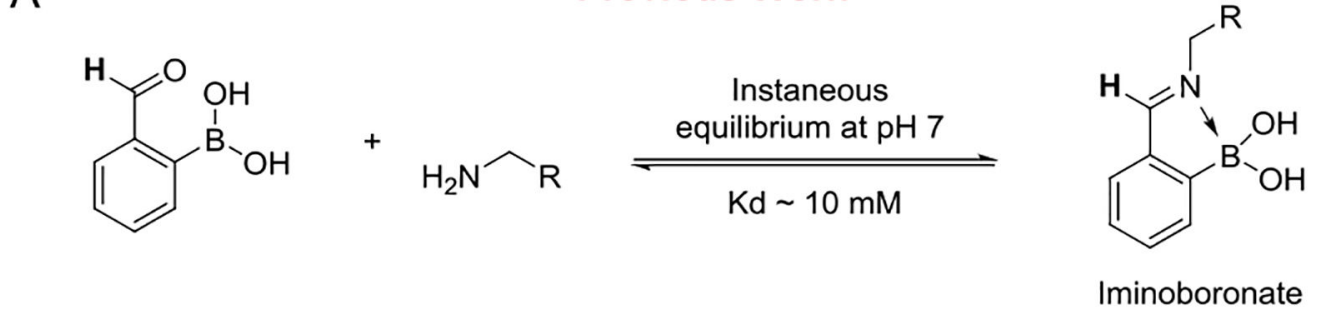

B<smiles>C/C(=C\B(O)O)C(=O)O</smiles>

This contribution<smiles></smiles>

Thiazolidino Boronate (TzB)

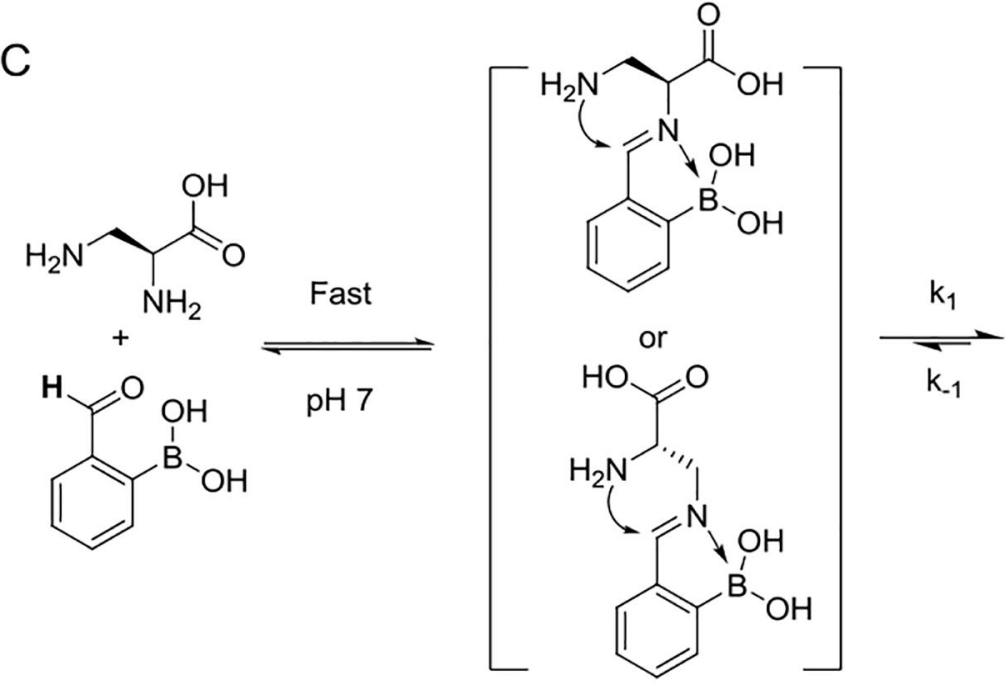<smiles>O=C(O)[C@@H]1CN2[C@H](C(=O)O)CN[C@@H]2c2ccccc2B1O</smiles>

Figure 1. Reversible conjugation chemistry of 2-formylphenylboronic acid.

A) Iminoboronate formation; B) TzB complex formation; C) IzB complex formation. 


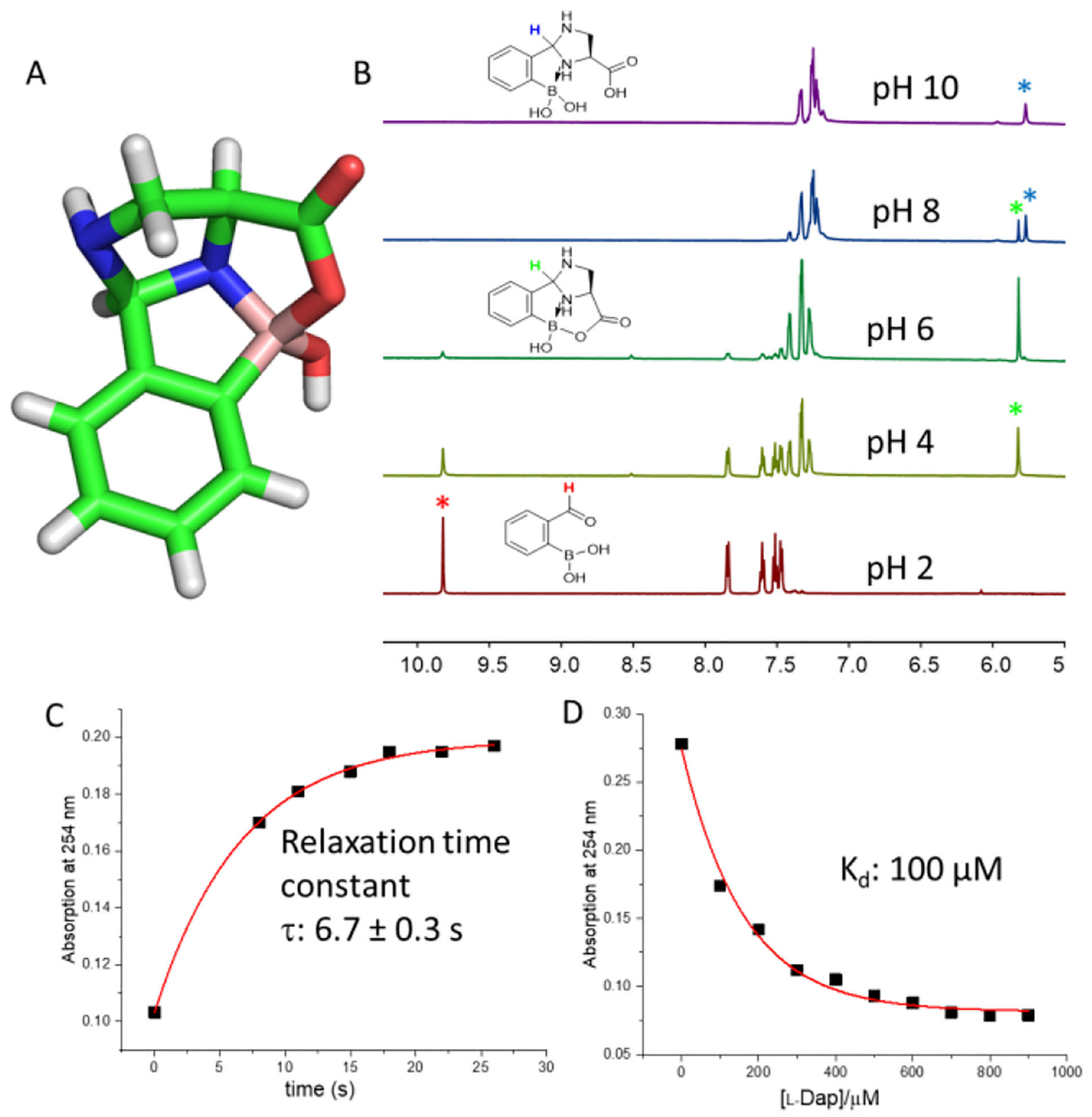

Figure 2. Characterization of an IzB complex formation by 2-FPBA and L-Dap.

A) Stick representation of the crystal structure of the IzB complex. Crystals were obtained from a methanol/water mixture at $\mathrm{pH}$ 7.4. C: green; $\mathrm{N}$ : blue; O: red; B: wheat; H: white. B) ${ }^{1} \mathrm{H}-\mathrm{NMR}$ spectra showing IzB conjugation at varied $\mathrm{pH}$. The colored $*$ denotes the proton of the same color in the structures. The samples were prepared with $10 \mathrm{mM}$ reactants in phosphate buffers of varied $\mathrm{pH}$. C) Dissociative relaxation kinetics of the IzB complex. The sample was prepared at $1 \mathrm{mM}$ and diluted 16.7 times for kinetic measurement. D) Titration (binding) curve of 2-FPBA with L-Dap, analysis of which yields the $K_{d}$ value. 2-FPBA was used at $50 \mu \mathrm{M}$ concentration. 

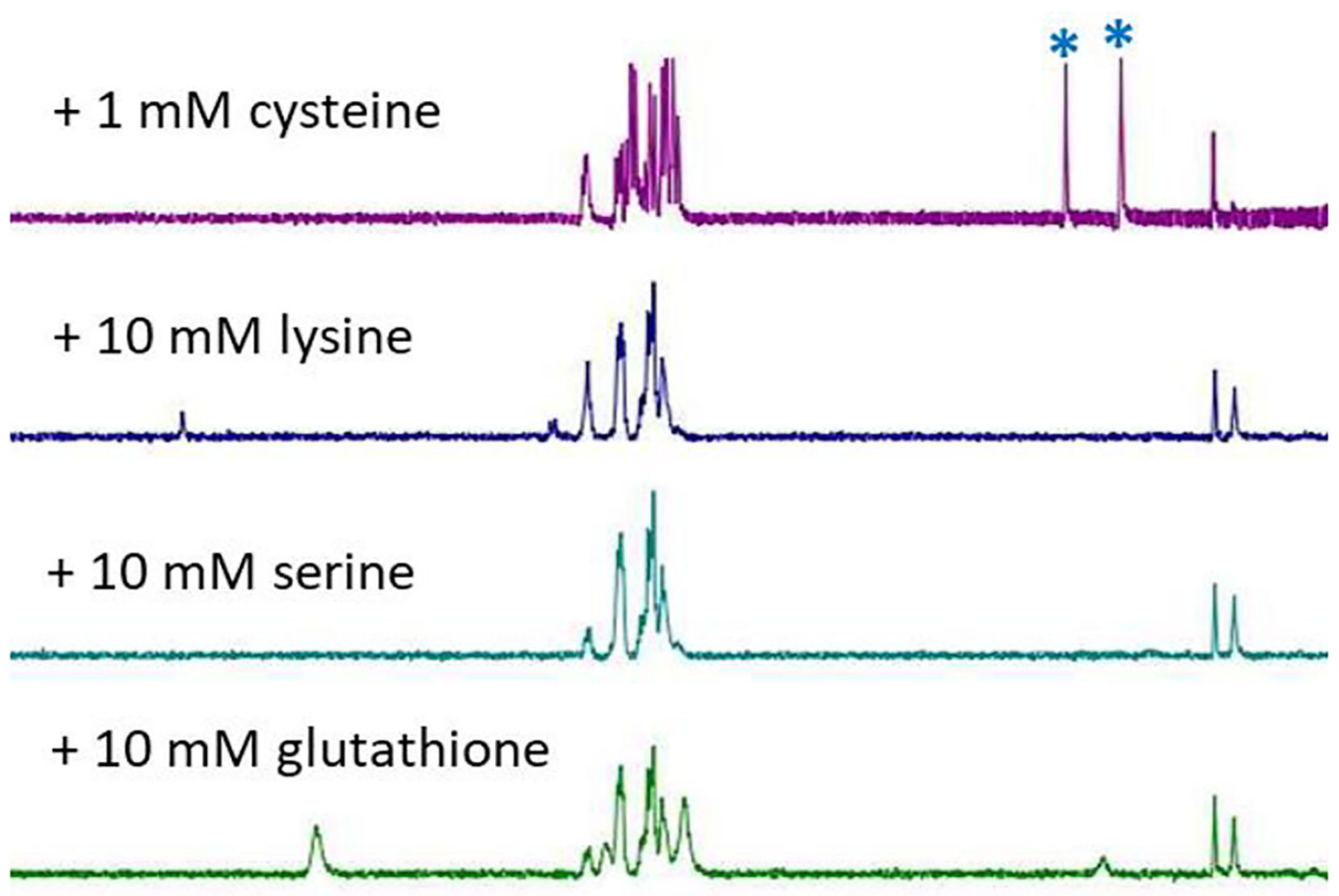

$+10 \mathrm{mM}$ glucose
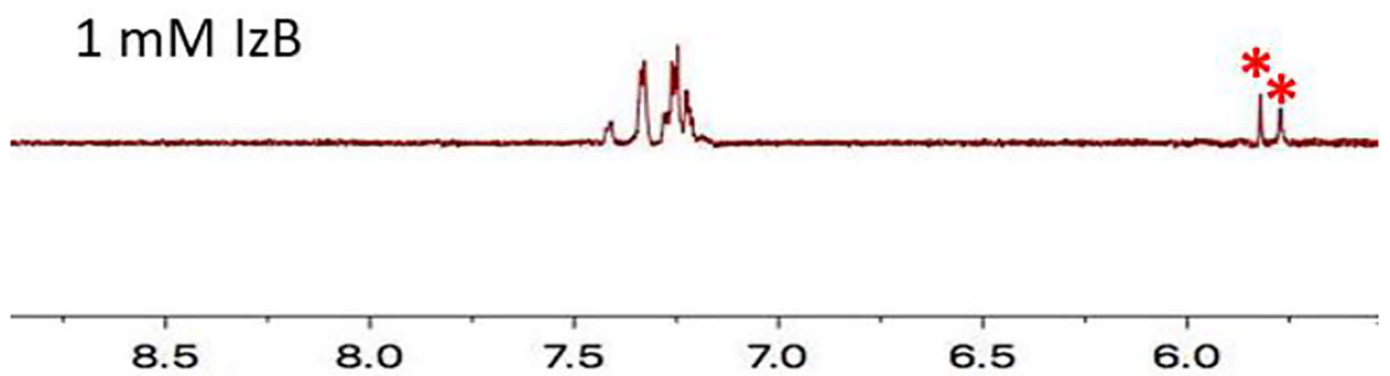

Figure 3. ${ }^{1} \mathrm{H}$-NMR analysis of the bioorthogonality of the IzB conjugation.

The ${ }^{1} \mathrm{H}$-NMR signature of the IzB complex remains unchanged with the addition of common metabolites except cysteine. * and * asterisks denote the signature peaks of IzB and TzB respectively. 

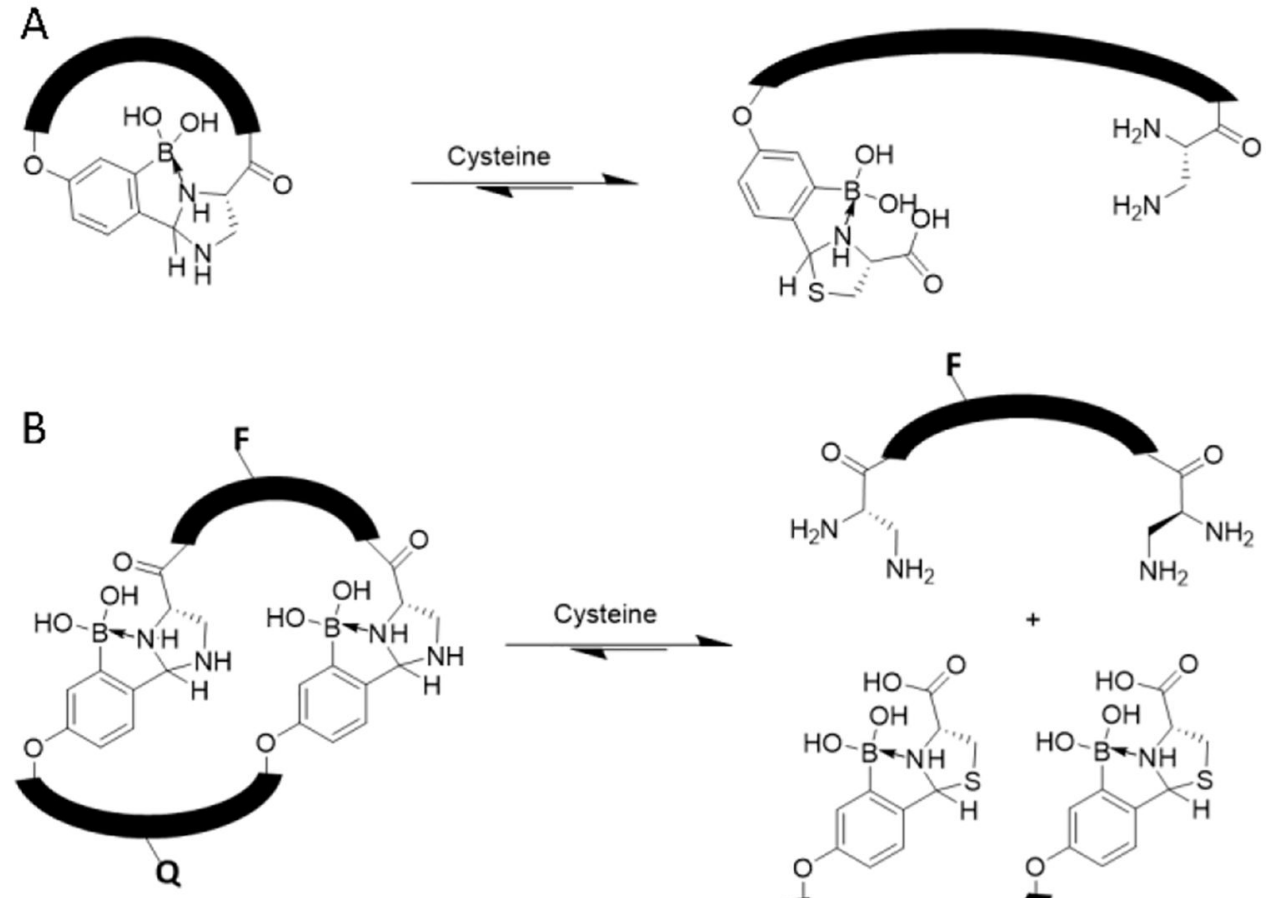

C
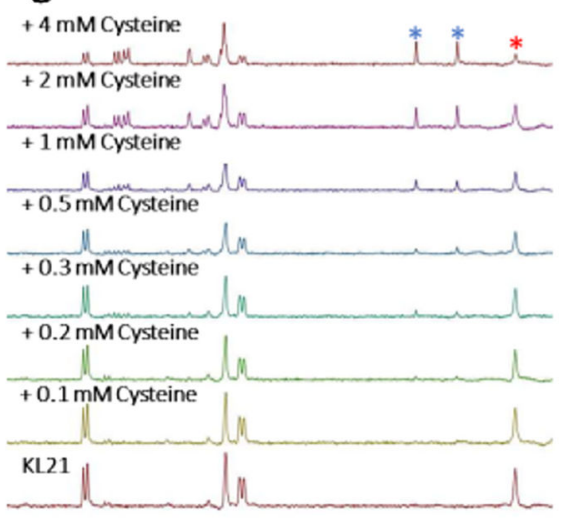

$\begin{array}{lllllllllll}7.5 & 7.3 & 7.1 & 6.9 & 67 & 6.5 & 6.3 & 6.1 & 5.9 & 5:\end{array}$

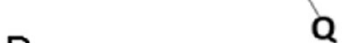

Q

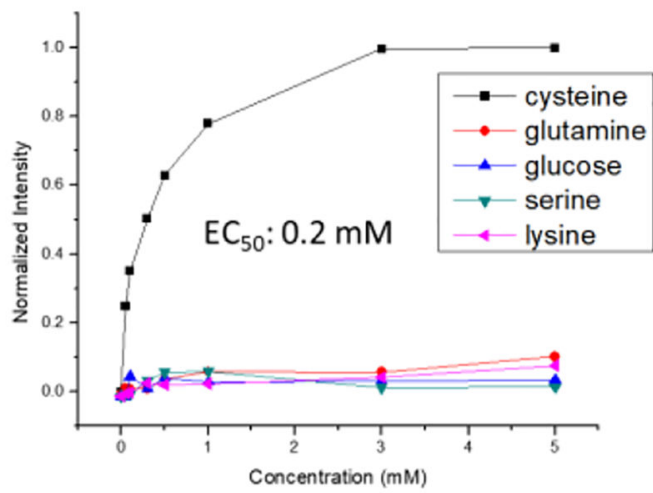

Figure 4. IzB cyclized peptides that respond to cysteine.

A) Scheme of cysteine responsive KL21 peptide linearization. B) Scheme of dual peptide fluorescence assay for cysteine detection. F represents the fluorophore (fluorescein) and Q represents the quencher (dabcyl). The full structure and synthesis is given in the Supporting Information. C) ${ }^{1} \mathrm{H}-\mathrm{NMR}$ spectra of the KL21 with increasing concentrations of cysteine revealing the IzB and TzB exchange. * and * denote the signature peaks of $\mathrm{IzB}$ and $\mathrm{TzB}$ respectively. D) Small molecule titration with quenched dual peptide IzB complex $(0.1 \mu \mathrm{M}$ KL22 + $1 \mu \mathrm{M}$ KL23) measuring fluorescence intensity. 